Mirza Mejdanija

\title{
La finis Austriae e gli ultimi racconti sveviani
}

Riassunto: Il 1914 è il mitico anno della finis Austriae ed è la data dell'inizio della catastrofe con cui si arriva all'ultima fase della decomposizione dell'Impero, solida base di un certo assetto borghese che comincia a vivere la situazione della crisi generale. Questa crisi è evidentemente anche il prodotto dei grandi processi di trasformazione della società borghese. Nel primo dopoguerra Svevo scrive il suo terzo romanzo che esce nel 1923 presso l'editore Cappelli. La coscienza di Zeno è ormai uno dei romanzi più importanti della letteratura italiana, anzi europea. Ma, proprio perché teso a denunciare l'indecifrabilità del reale, non può rispondere ai quesiti che solleva. Saranno invece i racconti successivi, redatti tra il 1923 e il 1928, a prendere le mosse proprio dai problemi irrisolti del terzo romanzo e a costruire il successivo passo in avanti della ricerca letteraria di Svevo. Ovviamente, questi dieci racconti rappresentano una fase intermedia tra il terzo e il 'quarto' romanzo, proponendo una diversa soluzione della crisi individuale. Quello che collega i 10 racconti sono l'impossibilità della pura teoria, l'ineluttabilità degli eventi, la metafora del vedere e quella della memoria.

\section{Finis Austriae and Svevo's last short stories}

Abstract: 1914 is the iconic year of finis Austriae and the beginning of the catastrophe, leading to the last phase of the disintegration of the Habsburg Empire which had provided a solid basis for a certain bourgeois order but was doomed to be hit by the general, widespread crisis. Needless to say, this crisis was also the result of major processes of transformation of the bourgeois society. In the first post-war period Svevo wrote his third novel La coscienza di Zeno. Published in 1923 with Cappelli publishers, it is now considered one of the most important novels in Italian, even European, literary history. However, precisely because it aims to denounce the indecipherability of reality, it cannot answer the questions it raises. Instead, Svevo's later short stories, written between 1923 and 1928, depart from the unresolved problems of the third novel and allow Svevo to make a step forward in his literary research. These ten short stories represent an intermediate phase between the third and 'fourth' novel, proposing a different solution

Ә Open Access. (C) 2020 Mirza Mejdanija, published by De Gruyter. (c))BY-NC-ND This work is licensed under the Creative Commons Attribution-NonCommercial-NoDerivatives 4.0 License.

https://doi.org/10.1515/9783110640069-012 
to the individual crisis. Common themes in the ten short stories are the impossibility of pure theory, the ineluctability of events, the metaphor of seeing and that of memory.

\section{Introduzione}

Svevo è l'interprete del periodo a cavallo tra l'Ottocento e il Novecento, epoca di passaggio in cui l'uomo e l'identità maschile entrano in crisi in seguito ai grandi processi di trasformazione della società borghese, fondata non più sul liberismo economico ma sul monopolio e caratterizzata dai problemi tipici di una società ormai di massa. Il declassamento dei ceti medi tradizionali, il ruolo dell'intellettuale che cambia per rispondere alle esigenze di una società molto più complessa e l'impossibilità di controllare una dinamica che investe in maniera occulta tutti i settori della vita pubblica e privata, fanno sì che l'individuo si senta insignificante all'interno di un nuovo ceto medio ridotto a massa anonima e amorfa. Alla fine dell'Ottocento l'Impero asburgico è travolto da una crisi che interessa diversi aspetti dell'assetto borghese, mentre l'inizio della Grande guerra sconvolge l'equilibrio politico europeo anche da un punto di vista di classe. Il 1914 è il mitico anno della finis Austriae e segna l'inizio della catastrofe che porta alla dissoluzione dell'Impero.

Nel primo dopoguerra Svevo scrive il suo terzo romanzo, pubblicato dall'editore Cappelli nel 1923, La coscienza di Zeno, uno dei più importanti della letteratura italiana, anzi europea, che, proprio perché intento a denunciare l'indecifrabilità del reale, non può rispondere ai quesiti che solleva. Saranno invece i racconti successivi, redatti tra il 1923 e il 1928, a prendere vita proprio dai problemi irrisolti del terzo romanzo e a far avanzare la ricerca letteraria di Svevo. Questi dieci racconti rappresentano una fase intermedia tra il terzo e il cosiddetto quarto romanzo, e propongono una diversa soluzione della crisi dell'individuo.

\section{La stagione dei racconti}

Tra il 1923 e 1928 Svevo è impegnato in quella che Tortora chiama «la grande stagione novellistica», ${ }^{1}$ componendo, per esempio, Corto viaggio sentimentale, Una

1 Massimiliano Tortora, Svevo novelliere, Pisa, Giardini, 2003, p. 76. 
burla riuscita e La novella del buon vecchio e della bella fanciulla. Dopo la pubblicazione del suo terzo romanzo, Svevo scrive dieci racconti in cinque anni, una produttività di gran lunga superiore rispetto a quella dei precedenti trentacinque anni di attività letteraria; e sono inoltre racconti molto più lunghi dei precedenti. Numerosi critici hanno applicato la categoria del 'non finito' all'ultima produzione sveviana, diversamente da Tortora, secondo il quale

\begin{abstract}
lo stato filologico dei racconti dunque non autorizza a indicare il 'non finito' come tratto preponderante della narrativa sveviana dal ' 23 in poi; le ragioni per cui tale interpretazione si è attestata vanno piuttosto cercate in quell'erroneo atteggiamento critico, troppo a lungo perpetrato, teso ad accorpare tutta la produzione narrativa susseguente La coscienza, debole nel differenziare la stagione dei racconti da quella del quarto romanzo, restio a riconoscere autonomia poetica alla novellistica sveviana. L'aver ritrovato tra le carte di Svevo un elevato numero di brani riconducibili a Il vegliardo, collocabili solo con estrema difficoltà in un discorso unitario, ha indotto molti studiosi a ritenere frammentario quasi tutto il materiale narrativo degli anni Venti. Ma nel momento in cui le pagine dei racconti vengono separate con la giusta fermezza da quelle del quarto romanzo, cessando di essere o schegge di un'enigmatica 'costellazione Zeno', o transitorie tappe di avvicinamento ad un progetto letterario successivo, non si può non constatare che i lavori novellistici compiuti sono diversi e senz'altro più numerosi da quelli ultimati dal più giovane Italo Svevo. ${ }^{2}$
\end{abstract}

In questo periodo, dunque, l'impegno di Svevo non è rivolto solo al teatro ma anche al racconto. Riesce a completare Proditoriamente, Una burla riuscita, Vino generoso, La madre, La novella del buon vecchio e della bella fanciulla, Argo e il suo padrone, La morte, Orazio Cima e L'avvenire dei ricordi, mentre lascia incompiuto, nonostante due revisioni, Corto viaggio sentimentale. Oltre a dedicarsi a questo ciclo di racconti si immerge in un'intensa attività editoriale, cui accompagna la promozione della Coscienza presso gli amici francesi (Larbaud, Crémieux e sua moglie Marie Anne Comnène) e italiani (Somarè, Montale e i solariani).

In quest'ultima produzione ritornano immagini della precedente, come pure commenti del narratore o di un personaggio:

Così ad esempio si nota che l'episodio biblico di Re David, a cui fu offerta in dono una giovane sunammita per aver salva la vita, è sfruttato per la prima volta in Corto viaggio sentimentale (1925-26), e poi ripreso ne La rigenerazione (1927) e ne La novella del buon vecchio (1927); la predilezione del signor Aghios per le mance contenute, consistenti in piccole somme, prelude a quell'abitudine degli anziani di concedere i soldi a rate, denunciata ne La novella; e si potrebbe citare ancora l'operazione di ringiovanimento, menzionata in Corto viaggio, ne Il vegliardo, e nucleo tematico e strutturale ne La rigenerazione; il complicato rapporto cane-catena, esemplificativa metafora della libertà umana, presente, limitandosi

2 Ivi, p. 15. 
alle opere di sicura datazione, in Vino generoso (1926-27) e in Corto viaggio sentimentale; e infine, per ricordare una delle pagine più celebri della narrativa sveviana, la sorpresa provata dal protagonista della Novella nel sentirsi dare del 'vecchio' dalla giovane amante non è dissimile da quella di Zeno di fronte alle parole di Felicita. ${ }^{3}$

Dal 1923 in poi Svevo è solito infatti utilizzare gli stessi frammenti narrativi, cambiati solo in minima parte, in testi cronologicamente vicini.

Dopo aver verificato la successione delle stesure dattiloscritte, Tortora mostra che i racconti sveviani, di ciascuno dei quali bisogna analizzare i temi trattati, possono essere datati solo in parte. Si può attribuire una data precisa a Proditoriamente (1923), La madre (1923-1924 e poi 1926), L'avvenire dei ricordi (1925), Una burla riuscita (1925-1926), Corto viaggio sentimentale (1925-1926), Argo e il suo padrone (1909-1915 e poi 1926), Vino generoso (1926-1927) e La novella del buon vecchio e della bella fanciulla (1927-1928). Sebbene non sia possibile indicarne l'esatto anno di composizione, risalgono al quinquennio 1923-1928 Orazio Cima e La morte. Il primo sembra risalire al 1927, mentre l'altro dovrebbe essere stato steso tra il 1925 e il 1928. Il filo rosso che lega i dieci racconti è costituito da alcuni temi ricorrenti, quali l'impossibilità della 'pura teoria' e l'ineluttabilità degli eventi, nonché dalla metafora del vedere e da quella della memoria.

\section{L’impossibilità della 'pura teoria'}

I racconti di questo quinquennio proseguono l'indagine epistemologica iniziata con La coscienza di Zeno e rivelano in modo sempre più esplicito l'impossibilità della 'pura teoria', cioè di teorizzare leggi universali e di presentare un sistema di comprensione definitivo. La molteplicità del reale spinge Svevo ad accentuare lo sforzo interpretativo richiesto ai suoi personaggi, ritenendo che se non è possibile definire l'ordine del mondo bisogna almeno provarci. L'unico approccio teorico possibile è indagare la realtà a posteriori, tentare di comprenderla; ogni tentativo di interpretare il mondo è accompagnato dalla consapevolezza che esso è precario e provvisorio. L'impossibilità della 'pura teoria' diviene più concreta nel racconto La madre, dove Curra, il pulcino protagonista, alla ricerca della madre inesistente, sogna l'amore, l'armonia e la completezza, ma riceve soltanto batoste. $\grave{E}$ un fallimento dal risvolto amaro: la nemica, cioè la presunta madre, si rivela es-

3 Ivi, pp. 43-44. 
sere solo la chioccia del vicino pollaio. Curra è triste perché non riesce a comprendere il reale e non per la consapevolezza di non poter ritrovare la madre immaginaria.

L'abisso che divide la teoria dalla realtà caratterizza anche La morte, in cui la medicina viene considerata incapace di capire fino in fondo il corpo umano:

Che cosa sapevano i medici? La malattia? Forse. Non l'organismo però, l'organismo di ogni singolo. E ricordò certi insegnamenti di Roberto. Gli uomini avevano tutti gli stessi organi e con quegli stessi organi componevano ognuno di essi un organismo originalissimo che mai prima era esistito. ${ }^{4}$

L'impossibilità della pura teoria è presente anche nel racconto Argo e il suo padrone. Un giorno un padrone legge sul giornale di quanto è accaduto in Germania, di un cane che ha cominciato a parlare la lingua umana, e così decide di insegnare l'italiano al suo cane Argo. All'inizio il cane è del tutto confuso e sconcertato, non capisce cosa voglia da lui il padrone. Ma poi è proprio Argo che inizia a raccontare tutto e a introdurre il lettore nel mondo dei cani. La visione del reale che propone l'eccentrico cane, nel contempo protagonista e narratore del racconto, è incentrata sull'io, è un punto di vista individuale e non ha come obiettivo la descrizione del mondo, che avrebbe invece un valore universale.

Argo preferisce dare delle chiavi di lettura dell'ambiente in cui si muove. Il fatto che le sue argomentazioni non valgano per qualsiasi situazione non gli importa. Le sue teorie sono limitate e precarie. Tutte le tesi di Argo partono dalla sua visione del mondo, in base alla quale è spinto a ricercare il piacere e a vivere serenamente, e a non preoccuparsi di comprendere tutti i fenomeni del mondo ma soltanto quelli che lo riguardano. Argo non sente il bisogno di rimediare all'abisso tra teoria e realtà, non lo percepisce come una malattia da cui guarire. Anzi, grazie proprio ai limiti e ai divieti posti dalla natura e dal padrone, riesce a orientarsi in un mondo troppo vasto. La vita di Argo è veramente 'originale' e la sua logica non arriva a spiegare buona parte degli eventi; quello del cane è perciò un approccio singolare e funzionale al magma e al caos del reale.

In Corto viaggio sentimentale Aghios, mentre cerca uno zolfanello, trova in tasca la busta con dentro le trentamila lire destinate a un pagamento da effettuare una volta giunto a destinazione. Il signore seduto di fronte a lui, vedendolo impegnato in una ricerca che sembra vana, racconta che sa sempre tutto quello che ha nelle tasche. Anche Aghios vorrebbe sapere sempre tutto quello che ha nelle tasche, le descrive come un armadio ambulante e dice di volerci mettere un registro che ne riporti la pianta e il contenuto. Il registro contenente la pianta delle

4 Italo Svevo, I racconti, Milano, Garzanti, 1985, p. 283. 
tasche è una delle tante metafore della 'pura teoria' e la prova di come il tentativo di dare ordine al reale sia destinato a fallire, poiché anche la pianta pensata per ritrovare facilmente gli oggetti si trova essa stessa nelle tasche. Non c'è via di scampo, l'onniscienza è fallace, la pretesa di conoscere tutto è illusoria. Ogni descrizione del reale non può che essere precaria e parziale. Il vecchio capisce che non è possibile definire l'ordine del mondo, prova quindi a dare un ordine al proprio vissuto, nonostante non venga fornita alcuna gerarchia definitiva degli oggetti e dei concetti. Non esistono regole rassicuranti, gli individui sono sempre costretti a ricorrere alle proprie interpretazioni per costruire un'unità che vorrebbe avere una legittimità universale ma che è invece condannata al livello del particolare:

Lo sforzo di catalogare è davvero inutile, se il pensiero che si sforza di risolvere in unità le contraddizioni del reale è destinato ad arrendersi e a constatare la sua avvenuta frantumazione. [...] È l'elogio del pensiero che sguscia, al punto di vanificare la ricerca sistematica del buon vecchio: l'ordine del logos appare inadeguato, la vita è nuda, un processo infiammatorio che a un dato momento ha fatto suppurare la materia morta, per poi tornare a cristallizzarsi, ridiventando frammento inorganico, inanimato e, per questo, puro, come la scrittura. [...] Non ci può essere allora gerarchia nella vita. ${ }^{5}$

Anche l'aneddoto del piccolo Pucci tremante per la paura di essere preso per un ladro rende chiara l'inattuabilità della pura teoria e mostra come un evento imprevedibile smentisca anche le certezze più fondate e rimetta in discussione ogni sistema faticosamente elaborato:

Poi raccontò che pochi giorni prima era con Pucci a passeggio e videro due carabinieri col loro mantello un po' minaccioso sotto a quel cappello napoleonico. E il bimbo spaventato domandò se quei carabinieri sapevano ch'essi non erano dei ladri. - Si può essere più sciocchi di così? - esclamò il Borlini.

Subito l'Aghios prese interesse al chiacchierio vuoto del suo compagno. Come si sentiva amico del piccolo Pucci dal cuore palpitante di paura d'essere preso per un ladro o forse di esserlo! Il ladro poteva essere preso in flagrante, ma non c'era una prova così risolutiva per il non ladro. Era come la prova Wassermann. La negativa non era mai sicura. Il microbo del furto poteva esserci nel sangue, ma aspettare una buona occasione per dar segno di vita. ${ }^{6}$

Anche nel racconto La novella del buon vecchio e della bella fanciulla la 'teoria' del vecchio non trova sviluppo. All'inizio il vecchio appare pieno di idee e

5 Cristina Benussi, La forma delle forme, Trieste, EUT, 2007, p. 209.

6 I. Svevo, I racconti, cit., p. 358. 
risoluto a scrivere il saggio, ma a un certo punto si blocca poiché incapace di risolvere un problema che si era posto. Scrive prefazione e primo capitolo velocemente, senza incontrare ostacoli e terminandoli in poco tempo. Nel capitolo decimo affiora la polemica sveviana nei confronti della pura teoria poiché il vecchio si rende conto dell'impossibilità di salvare il mondo dalla sua contingenza. Dunque la 'teoria' del vecchio non trova sviluppo, non per incapacità dell'autore ma per l'implicito fallimento che la stessa teoria comporta, poiché non si può descrivere scientificamente il mondo essendo questo un caos irrazionale. Il racconto termina con la morte del vecchio e il messaggio sotteso è che niente può essere detto a priori e in modo definitivo.

\section{L’ineluttabilità degli eventi}

In questi racconti è presente la riflessione sull'ineluttabilità degli eventi, che il più delle volte accadono indipendentemente dal volere dei personaggi. La vita è vittima della casualità che sfugge al controllo dell'individuo. Proprio questo è il motivo per cui non è possibile elaborare teorie atte a spiegare il caos del mondo, 'teoria' e 'realtà' non coincidono mai e l'individuo non riesce a costituirsi nella sua interezza una volta per tutte. L'ineluttabilità degli eventi è il tema centrale di Proditoriamente: Maier fallisce a causa dell'imprudente accordo commerciale stretto con il pericoloso imbroglione Barabich, per cui Maier decide di chiedere un aiuto economico all'amico Reveni, un prestito con cui riavviare la sua attività e rimediare all'errore commesso:

\footnotetext{
Tanti anni di onesta attività fortunata venivano annullati da un istante di spensieratezza! Non era ammissibile questo. Per allargare il proprio campo d'attività, il vecchio commerciante s'era lasciato indurre di firmare un contratto che lo metteva nelle mani di altre persone e queste persone dopo di aver sfruttato tutto il credito che da quella firma derivava loro erano addirittura scappate da Trieste non lasciando dietro di loro che pochi mobili di nessun valore. Il Maier aveva deciso di far fronte a tutti quegli impegni come il suo onore esigeva. Ma adesso gli pareva ingiusto di dover sottostare a quegl'impegni non suoi. Se il Reveni, notoriamente un buon uomo, accettava di addossarsene almeno temporaneamente una parte il suo destino si mitigava. Il Maier non ricordava di aver rifiutate delle proposte simili. Ricordava (e con grande chiarezza) di aver firmato quel contratto anche quello (così gli sembrava) una prova di fiducia nell'umanità, non ricordando che la prima idea di contrarlo era venuta dal desiderio di aumentare i suoi benefici. ${ }^{7}$
}

7 Ivi, pp. 291-292. 
Il fallimento finanziario divide la vita di Maier in due fasi distinte: quello che è successo prima dell'accordo con Barabich e quello che ne è conseguito. Un aiuto finanziario da parte di Reveni avrebbe potuto riportare Maier alla situazione antecedente al fallimento, tuttavia il disastro è avvenuto ancor prima dell'inizio del racconto e i due amici discutono su quanto è accaduto. Maier è quello che ha subito 'l'originalità della vita' ed è consapevole della sua precarietà, mentre Reveni è convinto del contrario. Reveni fa capire all'amico di essere a conoscenza del raggiro di cui è stato vittima, e in qualche modo lo rimprovera per essere stato così imprudente. Allora si finge malato, la moglie gli sta accanto tutto il tempo e lo aiuta a declinare la richiesta giunta dall'amico, mentre Maier cerca di convincerlo che la vita è imprevedibile e che quello che è successo a lui sarebbe potuto capitare a chiunque.

La vita è cinica e imprevedibile, tanto che mentre parlano Reveni è colpito da un improvviso e inatteso attacco cardiaco che gli spezza la vita spazzando via ogni certezza; Reveni muore e Maier vede svanire l'unica speranza di ricevere il necessario aiuto economico:

Egli uscì a passo di corsa. Non per far presto perché il Reveni non poteva oramai essere aiutato da nessuno ma per poter allontanarsi da quel cadavere.

E sulla via si ripeté la domanda: - Sta meglio lui od io? - Come era pacifico steso su quel sofà! Strano! Non si vantava più del proprio successo ingrandito dagli errori del Maier. Era rientrato nella generalità e da lì guardava inerte con quel bulbo protundente privo di gioia o di dolore. Il mondo continuava ma quell'avventura ne dimostrava l'intera nullità. L'avventura toccata al Reveni toglieva ogni importanza a quella toccata a lui. ${ }^{8}$

L'irreversibilità di quanto accaduto e i mutamenti di prospettiva che avvengono in determinati momenti testimoniano che la vita è imprevedibile, anzi, «"originale” se, come era accaduto per Zeno e Guido, l'inetto e il vincitore si scambiano i ruoli». ${ }^{9}$

Anche Una burla riuscita offre una singolare rappresentazione narrativa dell'imprevedibilità degli eventi che vanifica ogni teorizzazione a priori. Il protagonista subisce un inganno che non gli permetterà più di ritornare allo stato in cui viveva prima. Ancora più dell'orribile burla, il guadagno incassato inaspettatamente mostra l'immediato primo dopoguerra come una significativa allegoria dell'ineluttabilità degli eventi, conseguenza dell'incertezza sociale ed economica di quel periodo:

8 Ivi, p. 301.

9 C. Benussi, La forma delle forme, cit., p. 176. 
Strana vita quella dell'uomo, e misteriosa: con l'affare fatto da Mario quasi inconsapevolmente, s'iniziavano le sorprese del periodo postbellico. I valori si spostavano senza norma e tanti altri innocenti come Mario ebbero il premio della loro innocenza, o per tanta innocenza, furono distrutti; cose che s'erano viste sempre, ma parevano nuove perché si avveravano in tali proporzioni da apparire quasi la regola della vita. ${ }^{10}$

\section{La metafora del vedere e la metafora della memoria}

In questi racconti l'uomo vuole interpretare il mondo, caotico, magmatico e impenetrabile, e, per dirla con Tortora, si serve di due metafore: quella del vedere e quella della memoria. L'occhio e la capacità di osservazione sono i tratti che qualificano i personaggi di Malocchio e de La coscienza di Zeno, e nelle novelle successive al romanzo sono uno dei temi centrali della narrazione. La capacità di vedere e percepire la realtà diventa determinante poiché consente di conoscere, comprendere e interpretare meglio il reale, garantisce una visione più ampia del mondo, un punto di vista strategico e un'attenta osservazione. È appunto nelle novelle che si presenta maggiormente l'opposizione vedere/non vedere o visione/cecità tesa a caratterizzare il personaggio e a preparare il fallimento cui va incontro il ‘cieco’ protagonista.

Ricordare è un modo per vedere il passato e la ricostruzione degli eventi passati è sempre incompleta e transitoria, dato che dipende da come chi indaga è collocato nel tempo e nello spazio. Il passato ha un aspetto mutevole perché la persona che lo indaga non trova un'evoluzione lineare degli eventi ma solo sparsi frammenti che deve comporre in unità. La prospettiva del passato è condizionata in questo modo dal punto di vista soggettivo. La memoria si avvale della conoscenza e dell'interpretazione, è uno sguardo rivolto al passato che diviene una verità sottoposta a modifiche e aggiunte, ed è quindi una verità relativa:

Quello della modalità attraverso cui si fissa la memoria è uno dei temi più indagati negli anni che accompagnano la Coscienza, dove le immagini del passato, che Zeno sa di avere inventate, ma che non per questo sono false, ritornano, seppur non ad illuminare il presente. ${ }^{11}$

10 I. Svevo, I racconti, cit., pp. 269-270.

11 C. Benussi, La forma delle forme, cit., p. 177. 
Dopo aver raccontato le paure e i dubbi di Maier relativi alla possibilità di ricevere un prestito da Reveni, il narratore commenta:

\begin{abstract}
Vedeva appena allora, sembrava, ma non vedeva tutto, perché se tutto avesse visto avrebbe pur dovuto dire che lei o suo marito erano pronti a soccorrere o non volevano saperne.

Intervenne Reveni. Parve avesse inteso che la storia dovesse essere considerata proprio da un solo punto di vista, quello del povero suo amico. Stendendosi con un certo disagio sulla sua poltrona guardò in alto e brontolò: - Un brutto affare, un gran brutto affare! Sospirò e soggiunse guardando finalmente in faccia il Maier: - T’è toccata un'avventura ben brutta! Questo poi significava veramente che l'avventura era tanto brutta che nessuno ci pensava ad intervenire per renderla più sopportabile. Dunque niente soccorso e il Maier poteva esonerarsi dall'umiliarsi per domandarlo. ${ }^{12}$
\end{abstract}

Anche in Argo e il suo padrone è presente la metafora del vedere, solo che in questo caso la capacità di vedere è relativa a un cane:

Il mio cane di caccia, Argo, mi guardava con curiosità e un po' d'ansietà temendo che la mia irrequietezza non prendesse un'altra direzione. Anche lui sapeva riposare. Era accovacciato sul soffice tappeto sul quale poggiava anche il mento piatto, e l'unica parte irrequieta del suo corpo era l'occhio. ${ }^{13}$

In Una burla riuscita Mario è vittima della burla rozzamente congegnata da Gaia e ciò accade solo perché continua a 'non vedere' per quasi tutto lo svolgimento del racconto. La sua 'cecità' consiste nell'incapacità di collegare concretamente tutti gli elementi percepiti:

Si può dire che Mario non era un cattivo osservatore, ma che era, purtroppo, un osservatore letterario, di quelli che possono essere truffati col minimo sforzo, perché sanno fare l'osservazione esatta per deformarla subito a forza di concetti. Ora i concetti non mancano mai a chi ha un po' di esperienza di questa vita, dove le stesse linee e gli stessi colori si adattano alle più varie cose, che solo il letterato ricorda tutte. ${ }^{14}$

Nei racconti sveviani l'osservazione non è mai una mera percezione ma è sempre accompagnata dall'atto interpretativo. La verità non è lo svelamento dell'ignoto, ma piuttosto la costruzione di un senso, è il risultato che si ottiene passando attraverso la conoscenza e l'interpretazione. Solo grazie al mix equilibrato di questi due ingredienti si arriva alla vera percezione, altrimenti questa rimane inevitabilmente preclusa.

12 I. Svevo, I racconti, cit., p. 295.

13 Ivi, p. 122.

14 Ivi, pp. 239-240. 
Questo è proprio il dramma che si svolge nel racconto Una burla riuscita, dove il protagonista sogna continuamente il successo letterario e questo finisce per 'accecarlo' e indurlo a vedere gli eventi in modo deformato. Il povero Samigli ha visto ogni elemento della burla ma in ognuno di questi vuole trovare la conferma della vera esistenza del contratto editoriale. Solo quando il fratello lo mette in guardia comincia ad osservare gli eventi da una prospettiva diversa e i reali propositi di Gaia appaiono in tutta la loro evidenza. Il suo ritorno alla 'vista' non si concretizza nella percezione di elementi prima ignorati ma solo in un diverso ordinamento dei fatti:

I due fratelli stavano cenando insieme e fu sorprendente come dopo i primi bocconi presi con tutta calma, Mario ad un tratto, da solo, senza che nessuno gli avesse detto un'altra parola, si sentisse addirittura mancare scoprendo intera la burla. La scopriva con enorme sorpresa, e nello stesso tempo si sorprendeva di aver dovuto attendere una vaga parola d'avvertimento per saperla tutta. Aveva chiuso gli occhi apposta per non vedere e non intendere? Da bel principio egli aveva indovinato l'intima natura dei due messeri coi quali aveva avuto da fare e li avrebbe potuti smascherare subito quando in sua presenza i due svergognati s'erano abbandonati al riso. Perché non aveva pensato, perché non guardato? Ricordò ancora: sul naso affilato del tedesco gli occhiali avevano tremato per il riso trattenuto; un'oscillazione simile a quella di un motore su una vettura. Mario ebbe allora il pensiero tanto pronto e acuto che scoperse qualche cosa che dai suoi occhi era stato chiaramente percepito ma non ancora comunicato al suo cervello: quel pezzettino di carta tratto dal portafogli del tedesco, e che doveva scusare il riso cui i due compari s'erano abbandonati, era coperto di uno stampatello gotico. Gotico, tutto rette ed angoli. Ne era sicuro, come se lo vedesse allora. Perciò non poteva provenire da un postribolo di Trieste. Mentitori! E mentitori che gli avevano denotato il loro disprezzo non curandosi neppure d'essere accorti. ${ }^{15}$

Dunque Samigli aveva visto tutto ma viene a conoscenza della verità solo dopo aver messo da parte il sogno letterario e assunto un punto di vista più distaccato. L'ultimo dubbio si risolve quando Gaia, pestato a dovere da Samigli, confessa tutto:

Così, quando Mario incontra Gaia, lo prende a schiaffi e a calci, sicuro che le «vittorie dello spirito non v'ha dubbio, sono molto importanti, ma una vittoria dei muscoli è salutare assai». [...] La memoria va a quel suo primo racconto Una lotta, in cui lo sportman vinceva sul poeta, incapace di cogliere i segnali della sconfitta, tronfio com'era della presunta superiorità della poesia su ogni cosa. ${ }^{16}$

15 Ivi, pp. 259-260.

16 C. Benussi, La forma delle forme, cit., p. 223. 
La metafora del vedere, presente in numerose novelle, è il fulcro della riflessione sveviana sulla capacità del soggetto di conoscere, interpretare e dunque comprendere la realtà. È appunto l'immagine narrativa che illustra nel modo migliore come ogni teorizzazione, benché basata su dati oggettivi, sia relativa e parziale. L'osservazione presuppone infatti un punto di vista che è quello del soggetto calato nella stessa realtà contingente di cui intende offrire la spiegazione ultima e definitiva. Qualsiasi desiderio di superiorità, di guardare il mondo intero oggettivamente dal difuori, di vedere tutto chiaramente separato, non può che rivelarsi un'illusione, ingannevole e dannosa.

Guardare soggettivamente la realtà diventa l'unica via percorribile dall'io sveviano nell'opera Corto viaggio sentimentale e la bambina che comincia a piangere perché dal finestrino non riesce a vedere il treno né se stessa mentre viaggia è la prova che bisogna guardare il mondo dal di dentro. Non è possibile cogliere il reale nella sua interezza e il soggetto nell'atto stesso di guardare. Per questo la verità derivante da ogni teoria è una verità parziale, limitata e lacunosa.

Il modo in cui Orazio in Orazio Cima vede il reale dipende soprattutto dall'io, unico punto di riferimento: egli non cerca di descrivere il mondo né di determinare un valore universale, preferisce invece elaborare i valori dell'ambiente in cui si muove. Certo, le sue tesi non corrispondono al mondo che lo circonda, ma non se ne cura. Le sue teorie sono il frutto della sua visione del mondo. Orazio si impegna a vivere serenamente senza preoccuparsi di comprendere tutti i fenomeni del mondo che lo circonda perché gli basta tentare di capire quelli che lo riguardano.

L'avvenire dei ricordi è la storia di un vecchio che viaggia in Baviera e ricorda un analogo viaggio di tanti anni prima. Svevo vuole mostrare come il passato muti costantemente d'aspetto perché in certi momenti appaiono dettagli che dapprima non erano stati colti o erano stati dimenticati ma che fanno parte della nostra conoscenza. L'io che guarda indietro per ritrovare il proprio passato non segue uno sviluppo lineare delle vicende ma solo schegge e frammenti dispersi e instabili che deve comporre in un'unità. Improvvisamente il vecchio ricorda la bella moglie del direttore ma non riesce a capire se è l'immagine del primo giorno in cui l'ha vista oppure l'immagine rielaborata in seguito.

La concezione del tempo trascorso è legata al punto di vista del soggetto, a sua volta condizionato dalla situazione contingente in cui si trova. È il presente che guida a costruire il passato, un passato che si presenta tutt'altro che statico, anzi fluido e dai confini incerti. 


\section{Conclusione}

Nei racconti dell'ultimo quinquennio la figura dell'autore non si pone mai come garante della verità ma rappresenta una voce tra le voci e in questo modo mostra l'impossibilità della 'pura teoria' e la necessaria compresenza di più teorie a posteriori. Qualsiasi tentativo di catalogare e interpretare il mondo in modo univoco è vano, tanto che i racconti indagano solo particolari aspetti del reale e offrono interpretazioni parziali.

Questi racconti continuano la riflessione nata nell'ultimo capitolo della Coscienza di Zeno, in cui il rifiuto della psicoanalisi mostra la sconfitta della 'pura teoria' e rende illusorio qualsiasi tentativo di mettere a punto una definizione del mondo. I personaggi di questi racconti confermano l'insuccesso di ogni tentativo di redenzione da parte dell'individuo. Svevo incentra la sua attenzione sulle due cause che impediscono un sistema di comprensione universale: la 'pura teoria' è assolutamente fallimentare, vista l'impossibilità di assumere un punto di vista superiore, e gli eventi sono ineluttabili e imprevedibili. I racconti si differenziano dal terzo romanzo perché insieme alla rassegnazione per la frattura tra realtà e teoria c'è la fiducia di poter comunque esprimere certe idee e opinioni sul mondo.

I dieci racconti presentano una particolare fase dell'indagine letteraria sveviana, che inizia con l'ultimo capitolo della Coscienza di Zeno e offre gli strumenti necessari per la stesura de Il vegliardo. Questi non sono né il proseguimento del terzo romanzo né i materiali preparatori al quarto, poiché rappresentano una fase dell'indagine letteraria nettamente distinta dal terzo e quarto romanzo. 
